\title{
RESEARCH
}

Open Access

\section{Circular RNA circ-ZKSCAN1 inhibits bladder cancer progression through miR-1178-3p/ p21 axis and acts as a prognostic factor of recurrence}

Junming $\mathrm{Bi}^{1,2+}$, Hongwei $\mathrm{Liu}^{3+}$, Wei Dong ${ }^{4 \dagger}$, Weibin Xie ${ }^{1,2+}$, Qingqing $\mathrm{He}^{1,2}, \mathrm{Zijian} \mathrm{Cai}^{1,2}$, Jian Huang ${ }^{1,2^{*}}$ and Tianxin $\operatorname{Lin}^{1,2^{*}}$

\begin{abstract}
Background: Circular RNAs (circRNAs) represent a subclass of regulatory RNAs that have been shown to have significant regulatory roles in cancer progression. However, the biological functions of circRNAs in bladder cancer (BCa) are largely unknown.

Methods: Cell invasion models were established, and invasion-related circRNAs were detected by qPCR. Using above method, circ-ZKSCAN1 was picked out for further study. Circ-ZKSCAN1 expression and survival analyses were performed through GPCR. The survival curves were generated by the Kaplan-Meier method, and the log-rank test was used to assess the significance. Cell proliferation, migration and invasion were examined to investigate the function of circ-ZKSCAN1. Tumorigenesis in nude mice was assessed to determine the effect of circ-ZKSCAN1 in bladder cancer. Biotin-coupled probe pull-down assays, FISH and luciferase reporter assays were conducted to confirm the relationship between circ-ZKSCAN1 and microRNA. RNA-seq revealed different molecular changes in downstream genes.

Results: Here, we found that circ-ZKSCAN1 was downregulated in BCa tissues and cell lines. Circ-ZKSCAN1 levels were associated with survival, tumor grade, pathological T stage and tumor recurrence. Overexpressed circ-ZKSCAN1 inhibits cell proliferation, migration, invasion and metastasis in vitro and in vivo. Mechanistically, we demonstrated that circ-ZKSCAN1 upregulated p21 expression by sponging miR-1178-3p, which suppressed the aggressive biological behaviors in bladder cancer.

Conclusions: These results reveal that Circ-ZKSCAN1 acts as a tumor suppressor via a novel circ-ZKSCAN1/ miR-1178-3p/p21 axis, which have the important role in the proliferation, migration and invasion ablitities of $\mathrm{BCa}$ cells and provide a novel perspective on circRNAs in BCa progression.
\end{abstract}

Keywords: Circ-ZKSCAN1, Bladder cancer, miR-1178-3p, p21

\footnotetext{
* Correspondence: urolhj@sina.com; lintx@mail.sysu.edu.cn

†unming Bi, Hongwei Liu, Wei Dong and Weibin Xie contributed equally to this work.

1'Department of Urology, Sun Yat-Sen Memorial Hospital, Sun Yat-Sen

University, 107.W. Yanjiang Road, Guangzhou, Guangdong 510120, People's

Republic of China

Full list of author information is available at the end of the article
}

(c) The Author(s). 2019 Open Access This article is distributed under the terms of the Creative Commons Attribution 4.0 International License (http://creativecommons.org/licenses/by/4.0/), which permits unrestricted use, distribution, and reproduction in any medium, provided you give appropriate credit to the original author(s) and the source, provide a link to the Creative Commons license, and indicate if changes were made. The Creative Commons Public Domain Dedication waiver (http://creativecommons.org/publicdomain/zero/1.0/) applies to the data made available in this article, unless otherwise stated. 


\section{Background}

Bladder cancer $(\mathrm{BCa})$ is one of the most common urinary malignancies, with approximately 429,800 new cases and 165,100 deaths annually worldwide [1]. BCa is categorized into 2 groups: non-muscle-invasive bladder cancer (NMIBC) and muscle-invasive bladder cancer (MIBC) [2]. NMIBC is the most common $\mathrm{BCa}$ and is often treatable [2]. Despite multiple therapeutic approaches have improved, the prognosis for MIBC with extensive invasion and metastasis remains poor [3]. To metastasize, BCa cells must undergo a series of selective steps, including transformation, migration, invasion, vascularization, transportation within the circulatory system, and establishment in different organs [4]. Among these highly selective events, the proliferation, migration and invasion of BCa cells into muscle are crucial early steps [4]. However, the mechanisms of proliferation, migration and invasion have not been fully elucidated. Thus, to improve the treatment of bladder cancer, it is vital to have a better understanding of changes in genes during proliferation, migration and invasion.

Circular RNAs (circRNAs) make up a novel class of regulatory RNAs that are formed by a covalently closed loop $[5,6]$. With massively parallel sequencing technology, researchers were able to explore the functions of circRNAs [7]. Previous studies revealed that the expression of circRNAs is cell type- and tissuespecific and can be mostly independent of the host gene [8]. CircRNAs are involved in the progression of many cancers, such as gastric cancer, glioma, and hepatocellular cancer, by being sponges of microRNAs and keeping target genes away from miRNAs [9-11]. Nevertheless, only a few circRNAs are predicted to be able to carry out such "sponging" activities [12]. Moreover, previous studies showed that circRNAs was more stable than mRNAs [9-11]. Besides, some circRNAs could have the potential to be biomarkers or prognostic factors in tumors [13]. However, few circRNAs in $\mathrm{BCa}$ have been reported [14, 15]. The functions of circRNAs in BCa remain largely unknown and need to be elucidated.

MicroRNAs (miRNA), a class of small and non-coding RNA, directly regulate a variety of biological processes of mRNAs in multiple cancer types [13]. Increasing evidences demonstrated that miRNAs exert influences on inhibiting or promoting invasion, migration and apoptosis in bladder cancer [16, 17]. For instance, miR-520f could reverse EMT and inhibit metastasis by targeting ADAM9 and TGFBR2 [18]. Mir-129 could exert growth inhibitory effect and induce cell death through targeting GALNT1 and SOX4 [19]. Recently, studies have reported that circRNAs could act as "miRNA sponges" in many cancers [20]. However, the relation between circRNAs and miRNAs has not been clearly elucidated in bladder cancer.
In the present research, we identified circRNAs from previous circRNA microarray data of human $\mathrm{BCa}$ tissues and normal bladder tissues [21]. Then we established highly invasive (UM-UC-3-M and T24-M) and lowly invasive cell sublines (UM-UC-3-NM and T24-NM) using a repetitive transwell assay in vitro [21]. We examined the circRNAs expression profile of each cell subline to identify differentially expressed circRNAs related to $\mathrm{BCa}$ invasion. Among these, circ-ZKSCAN1, a significantly upregulated circRNA in lowly invasive cells, was shown to be closely correlated with $\mathrm{BCa}$ tumorigenesis and metastasis in patients. Furthermore, we found that enforced circ-ZKSCAN1 could suppress $\mathrm{BCa}$ progression in vivo and in vitro through a novel circ-ZKSCAN1/miR-11783p/p21 axis.

\section{Methods}

\section{Tumor specimens}

$\mathrm{BCa}$ tissues and the matched normal tissues were obtained from the bladder cancer tissue bank of our institute. All patients were histopathological diagnosed with $\mathrm{BCa}$ and undergone radical cystectomy between 2013 and 2017, without neoadjuvant chemotherapy or radiotherapy. Sixty-eight pairs of samples were frozen in liquid nitrogen and extracted by trizol. The grading of BCa was determined according to the histologic tumor grading system of the World Health Organization [22], and $\mathrm{BCa}$ staging was examined according to the TNM Classification of Malignant tumors established by the Union for International Cancer Control [23]. The study was approved by the ethical Review Committee of Sun Yat-sen Memorial Hospital. All the patients had signed the informed consent. Additionally, a cohort of $68 \mathrm{BCa}$ patients with histopathological diagnosis were followed up. Patients were followed up till February 2018 and the median follow-up time is 81.3 months.

\section{Cell culture and transfection}

Human Bladder cancer cell lines (T24, UM-UC-3, 5637, EJ, SV-HUC-1) were purchased from ATCC (American Type Culture Collection. Manassas, VA, USA). T24, 5637, EJ and SV-HUVEC Cells were cultured in RPMI-1640 (Gibco, Shanghai, China). While UM-UC-3 was cultured in DMEM (Gibco, Shanghai, China). All the medium was supplemented with $10 \%$ fetal bovine serum and $1 \%$ penicillin/ streptomycin (Gibco, Shanghai, China). Highly metastatic and lowly metastatic BCa cell model were established by the method described by previous study [21]. Eventually, highly invasive cell clones UM-UC-3-M, T24-M, and a lowly invasive clones UM-UC-3-NM, T24-NM were established. The cells were grown in a humidified atmosphere of $5 \% \mathrm{CO} 2$ at $37^{\circ} \mathrm{C}$. siRNAs and microRNA mimics were purchased from GenePharma (Shanghai, China). Oligos were showed in Additional file 1 Table S1. Transfection 
was carried out using reagent Lipofectamine RNAiMAX (Thermo Fisher Scientific Inc. Massachusetts, USA) following manufacturer's protocol.

\section{CircRNA plasmid construction and stable transfection}

To construct circ-ZKSCAN1-overexpressing plasmids, circ-ZKSCAN1 cDNA was synthesized and cloned into the plenti-ciR-GFP-T2A vector (IGE Biotech Co, China). Plasmids were transfected into 293 Tcells to package lentivirus using X-treme (Sigma, USA) according to the manufacturer's instructions. T24 and UM-UC-3 cells were infected with the packaged lentivirus and selected with $2 \mu \mathrm{g} / \mathrm{ml}$ puromycin for 3 days.

\section{RNA isolation and quantitative real-time PCR}

Total RNA was isolated using the RNAiso Plus (TaKaRa, Japan) according to the manufacturer's instructions. cDNA was synthesized using the PrimeScript RT Reagent Kit (Takara, China) and microRNA First-Strand cDNA Synthesis Kit (Sangon Biotech, China). The expression of circRNA and mRNA in cancer samples and cell lines was performed on an ABIPRISMVR 7300 Sequence Detection System (Applied Biosystems). Each reaction was performed in triplicate. Additional file 1 Table S1 lists the specific primers.

\section{Western blotting}

Western blotting was performed as previously described [24]. Primary antibodies specific to p21 (1:1000; CST, Danvers, Massachusetts, USA) and GAPDH (1:5000; CST, Danvers, Massachusetts, USA) were used. The blots were then incubated with goat anti-rabbit or anti-mouse secondary antibody (CST) and visualized by commercial ECL kit (Pierce, Rockford, IL).

\section{MTS assay}

BCa cells (1000 cells per well) were seeded into 96-well plates and $20 \mu \mathrm{l}$ MTS (Promega, Beijing, China) was added to each well for $3 \mathrm{~h}$ incubation. And then we measured the absorbance of each well at $492 \mathrm{~nm}$ every $24 \mathrm{~h}$ for 6 times.

\section{Cell migration and invasion assay}

For cell migration assays, cell suspensions $\left(5 \times 10^{4}\right.$ cells) were seeded to the upper chamber and incubate for $20 \mathrm{~h}$ with $1 \%$ FBS. For invasion assays, the bottom of the upper chamber was coated with Matrigel (BD Bioscience) and incubate for $24 \mathrm{~h}$.

After the incubation, cells were fixed and stained with crystal violet. Through counting the cells under the microscope, migration and invasion rates were quantified.

\section{Animal experiments}

All animal experiments were approved by the the ethical Review Committee of Sun Yat-sen Memorial Hospital. 4 weeks old Male BALB/c nude mice were purchased from the Experimental Animal Center, Sun Yat-sen University (Guangzhou, China). Mice were randomly divided into two groups: vector and circ-ZKSCAN1. Cells $\left(5 \times 10^{6} /\right.$ mice $)$ were injected into the foot-pad of right hind or subcutaneously injected into upper back of nude mice. On day 28 , the mice were euthanized and primary tumors and popliteal lymph nodes were enucleated and paraffin embedded.

\section{Immunohistochemistry analysis}

All paraffin-embedded tissue sections were examined by immunohistochemistry (IHC) using the experimental procedure as previously described with antibody specific for p21 (1:200; CST, Danvers, Massachusetts, USA). At least two pathologist had assessed the immunoreactivity in each section.

\section{CeRNA analysis and target prediction}

Using the bio-informatic database miRanda and TargetScan, we predicted the miRNA-binding sites of circ-ZKSCAN1. The interaction between mRNA and miRNA was predicted by miRanda and TargetScan. Filtering restrictions were as follows: Context Sore $\geq 90$.

\section{Pull-down assay}

The pull-down assay was performed using the procedure as previously described $[14,15]$. In brief, T24 cells that stably expressed circ-ZKSCAN1 were harvested, lysed, and sonicated. To generate the probe coated beads, circ-ZKSCAN1 probe and NC probe was incubated with magnetic beads. After $2 \mathrm{~h}$ incubation, cell lysates were incubated with the probes overnight. After the incubation, the bound RNAs were washed and purified for the analysis. CircZKSCAN1 biotinylated-probe was designed and synyhesized by GenePharma (Shanghai, China).

\section{Fluorescence in situ hybridization (FISH)}

The Fluorescence in situ hybridization (FISH) assay was performed using the procedure as previously described [25]. In brief, T24 cells were seeded and fixed in confocal dish. After prehybridization and hybridization, cells were incubate with cy3-labelled circ-ZKSCAN1 probe (GenePharma, China) at $37^{\circ} \mathrm{C}$ overnight. For double FISH assay, circ-ZKSCAN1 overexpressed T24 cells were transfected with miR-1178-3p mimics. After the transfection, cells were hybridized with circ-ZKSCAN1 probe (cy3-labelled) and miR-1178-3p probe (cy5-labelled). The images were acquired on ZEISS LSM800 Confocal Microscope (Carl Zeiss AG, Germany). Sequences of probes were showed in Additional file 1 Table S1. 


\section{Luciferase assay}

Dual-luciferase reporter assay was used to evaluate the direct binding between circ-ZKSCAN1 and miRNAs. psiCHECK2 (GenePharma, China) vector contains firefly luciferase gene (hLuc+) and renilla luciferase gene (hRluc). The sequence of circ-ZKSCAN1 was cloned into the vector. NC vector or circ-ZKSCAN1 vector was co-transfected with each miRNAs mimics. The relative values of hLuc+ and hRluc were detected by Centro LB960 XS3 (Berthold, German). Luciferase reporter assay was used to detect whether p21 is the direct target of miR-1178-3p. The 3'UTR sequence of p21 was cloned into pcDNA3.0 vector. Next, miR1178-3p mimics or NC was co-transfected with wildtype vector or the mutant vector. The relative value of luciferase was also detected by Centro LB960 XS3 (Berthold, German).

\section{Statistical analysis}

Statistical analysis in our cohorts was carried out with Graphpad prism. The Chi-square test was used to evaluate the association of the expression of circZKSCAN1 with the patient's clinicopathological characteristics. Survival curves were assessed with the KaplanMeier method and compared by the log rank test. Correlations were analyzed by Pearson's correlation test. $P$ values of $<0.05$ were considered significant. The data were presented as means \pm Standard Deviation
(SD) in the bar charts and were calculated difference by either Student's t-test or Chi-square test. P value of < 0.05 was considered statistically significant.

\section{Results}

Identification of invasion-related circRNAs in BCa cells

First, we analyzed and selected 35 differentially expressed circRNAs from circRNAs microarray data of previous study (fold change $\geq 2.0$ or $<0.5$ and $P<0.05$ ) [25]. Using qPCR, 21 circRNAs were detected in BCa cell lines. (Additional file 2 Table S2). To determine the mechanism of $\mathrm{BCa}$ migration and invasion, we established highly invasive $\mathrm{BCa}$ cell lines and lowly invasive $\mathrm{BCa}$ cell lines using the repeated invasion transwell method (Fig. 1a) [21]. Using this method, we established two lowly invasive sub-clones T24-NM, UMUC-3-NM, and two highly invasive sub-clones T24-M, UMUC-3-M (Fig. 1b). Twwenty-one circRNAs were detected in sub-clone cell-lines by qPCR. Fourteen circRNAs were differentially expressed in sub-clone cell-lines by qPCR and found to have effects on invasive abilities of $\mathrm{BCa}$ cell lines by transwell migration assays (data not shown).

\section{Characterization of circ-ZKSCAN1 in BCa cells}

According to the result of qPCR, circRNA_0001727 was significantly upregulated in T24-NM and UM-UC-3-NM. Then, we focused on its clinical characteristics, functionalities, and mechanisms for further study. CircRNA_0001727, termed circ-ZKSCAN1, was upregulated 2.73-fold in T24-

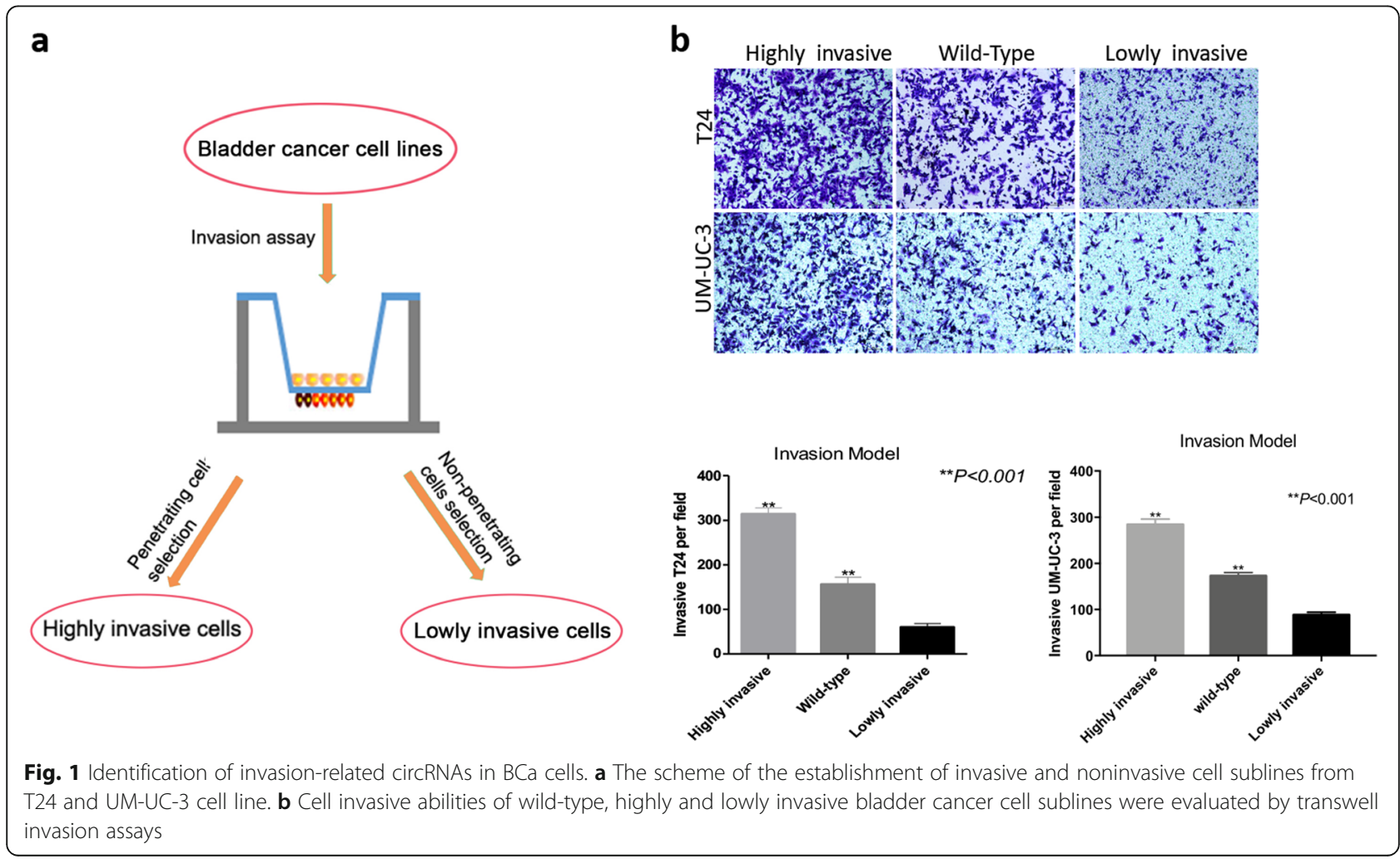


NM and 3.32-fold in UM-UC-3-NM (Fig. 2a). Additionally, circ-ZKSCAN1 showed lower expression in 4 BCa cell lines (5637, UM-UC-3, T24, EJ) than in the normal urothelial cell line (SV-HUC-1) (Fig. 2b). Furthermore, through nuclear mass separation assay and $\mathrm{FISH}$, we found that circ-ZKSCAN1 was predominantly located in the cytoplasm (Fig. 2c, d). Taken together, these results indicate that circZKSCAN1 is downregulate in $\mathrm{BCa}$ and mainly located in cytoplasm.

\section{Circ-ZKSCAN1 expression was decreased in BCa and associated with clinical characteristics}

To investigate whether circ-ZKSCAN1 was involved in $\mathrm{BCa}$ progression, we detected and analyzed circ-ZKSCAN1 expression in two independent cohorts of $\mathrm{BCa}$ tissues and matched adjacent normal tissues. We first performed a qPCR analysis of a 68-cases Cohort 1 of $\mathrm{BCa}$ tissues and matched adjacent normal tissues. Compared to the adjacent normal tissues, circ-ZKSCAN1 expression was significantly downregulated in the BCa tissues (Fig. 3a). While the data from TCGA and Cohort 1 showed that there was no significant change in ZKSCAN1 mRNA between BCa tissues and matched adjacent normal tissues (Additional file 3 Figure S1a and b).
To further investigate whether circ-ZKSCAN1 expression was correlated with the clinical features of $\mathrm{BCa}$ and patient prognosis, we examined a 137-cases Cohort 2 with complete survival data and clinical characteristic by qPCR. Remarkably, clinicopathological correlation analysis revealed a negative correlation between circ-ZKSCAN1 levels and recurrence (Fig. 3b). Moreover, statistical analysis revealed that patients with high grade, advanced pathological $\mathrm{T}$ stage (Fig. 3c) and positive lymphatic metastasis had lower expression of circ-ZKSCAN1 (Table 1). However, there was no correlation between circ-ZKSCAN1 expression and tumor size. Furhermore, Kaplan-Meier survival analysis showed that patients with low circ-ZKSCAN1 expression were significantly associated with poorer overall survival and disease free survival (Fig. $3 \mathrm{~d}$ and e). These findings clearly demonstrate that circ-ZKSCAN1 is downregulated in $\mathrm{BCa}$ and lower circ-ZKSCAN1 expression may lead to a poor prognosis for $\mathrm{BCa}$.

\section{Enforced expression of Circ-ZKSCAN1 inhibited} proliferation, migration, and invasion of $\mathrm{BCa}$ cells in vitro Loss-of-function and gain-of-function assays were performed to evaluate whether circ-ZKSCAN1 could affect the biological behavior of BCa cells. Since T24 and

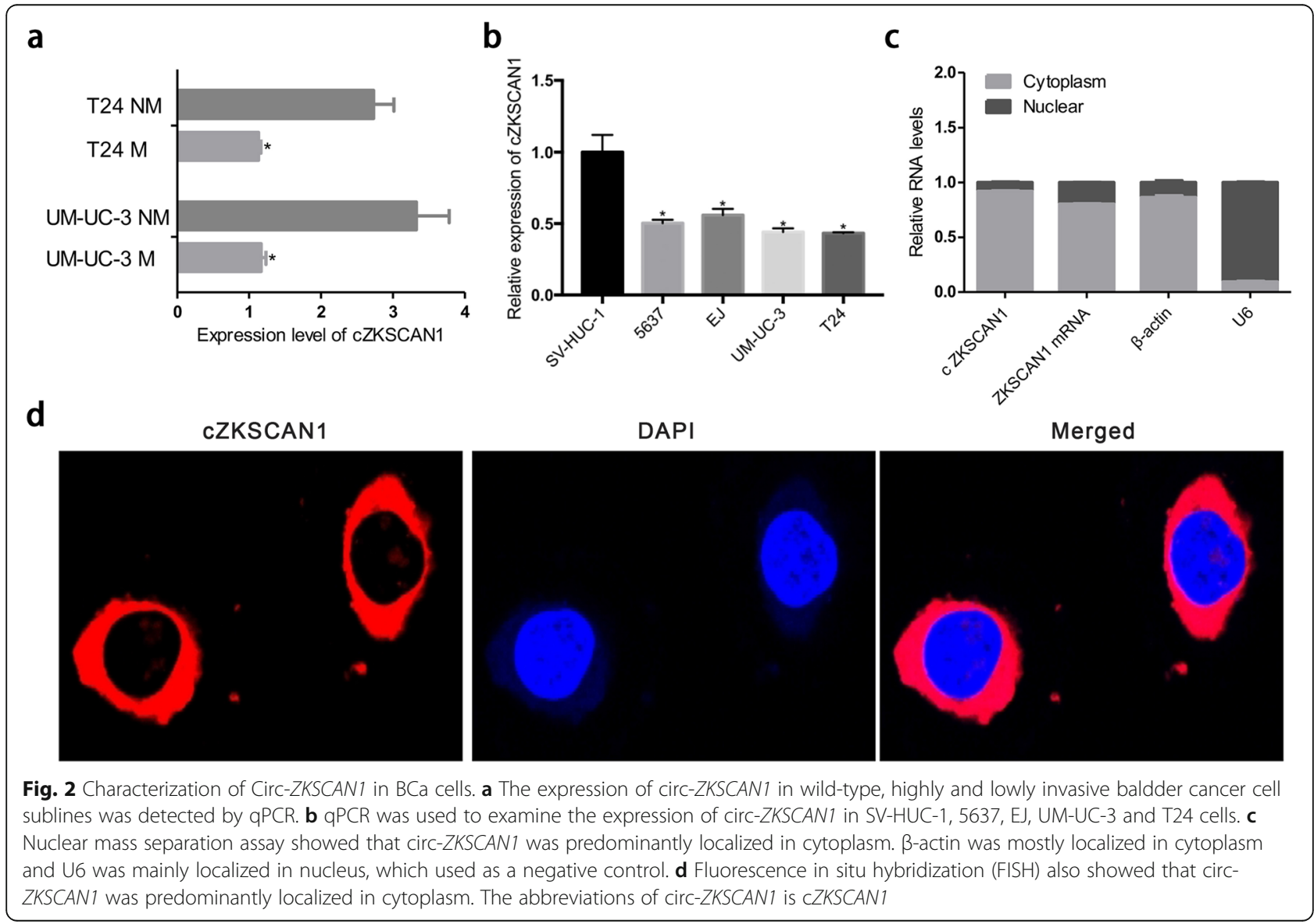




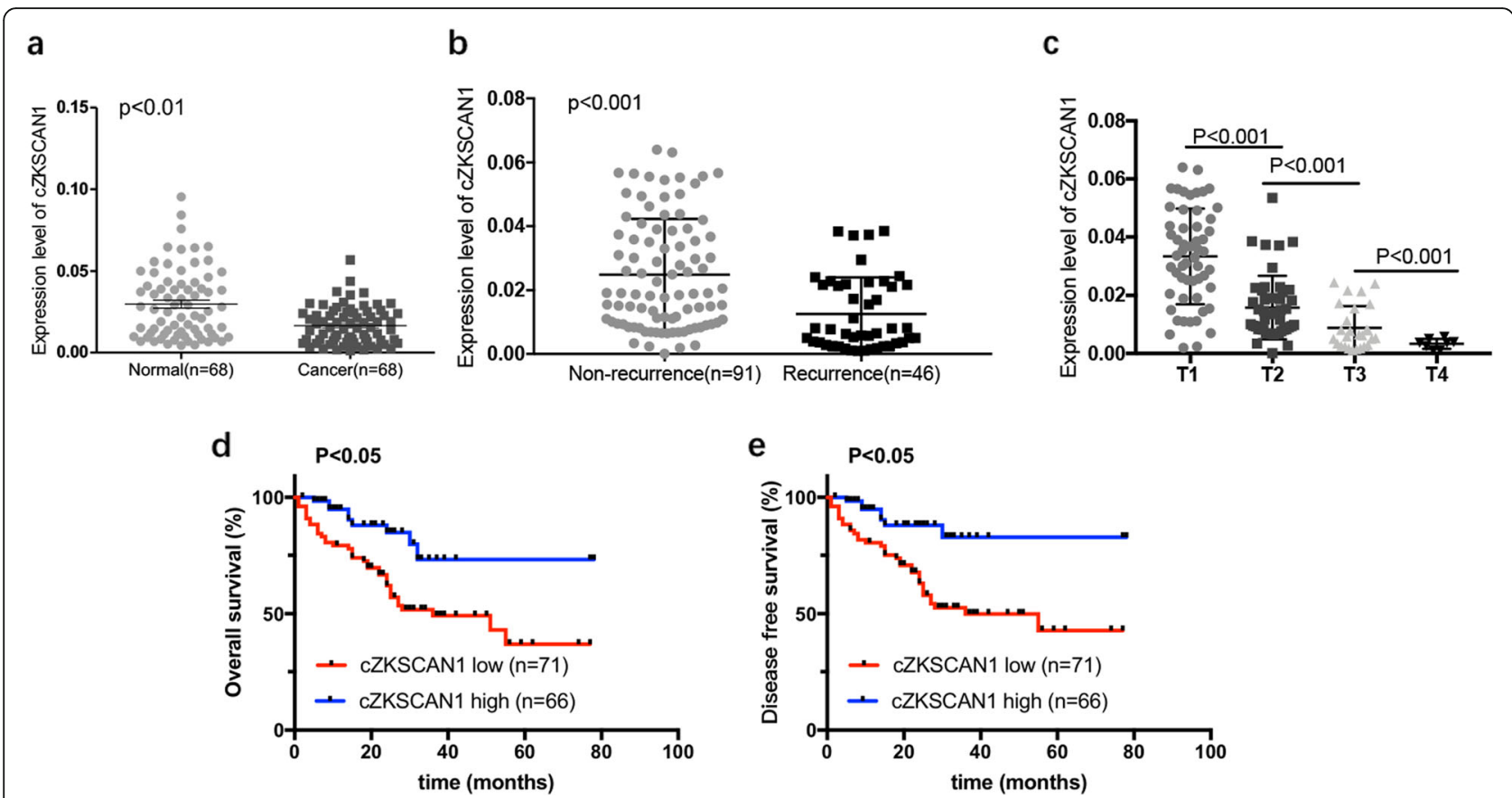

Fig. 3 Circ-ZKSCAN1 expression was decreased in BCa and associated with clinical characteristics. a qPCR was used to examine the expression of circ-ZKSCAN1 in 68 pairs of BCa and normal bladder tissues. $\beta$-actin was used us internal control. b Expression of circ-ZKSCAN1 was significantly lower in recurrence patients. c Advanced T stage is associated with lower circ-ZKSCAN1 levels. d Kaplan-Meier survival plots showed that lower expression of circ-ZKSCAN1 leaded to poorer prognosis. e Disease-free survival revealed that lower expression of circ-ZKSCAN1 was associated with shorter time

Table 1 Correlations between circ-ZKSCAN1 expression levels and clinicopathological characteristics in $\mathrm{BCa}$

\begin{tabular}{|c|c|c|c|c|}
\hline \multirow[t]{2}{*}{ Characteristics } & \multirow[t]{2}{*}{ Case } & \multicolumn{2}{|c|}{ Circ-ZKSCAN1 expression } & \multirow{2}{*}{$\begin{array}{l}P \\
\text { value }\end{array}$} \\
\hline & & Low (71) & High (66) & \\
\hline Age (years) & & & & 0.1248 \\
\hline$<65$ & 69 & 31 & 38 & \\
\hline$\geq 65$ & 68 & 40 & 28 & \\
\hline Gender & & & & 0.1077 \\
\hline Male & 115 & 56 & 59 & \\
\hline Female & 22 & 15 & 7 & \\
\hline Pathology stage & & & & 0.0001 \\
\hline pTa-pT1 & 55 & 11 & 44 & \\
\hline pT2-pT4 & 82 & 60 & 22 & \\
\hline Histological grade & & & & 0.0001 \\
\hline Low & 19 & 1 & 18 & \\
\hline High & 118 & 70 & 48 & \\
\hline Tumor size (cm) & & & & 0.8037 \\
\hline$<3$ & 119 & 61 & 58 & \\
\hline$\geq 3$ & 18 & 10 & 8 & \\
\hline Lymph nodes status & & & & 0.0038 \\
\hline Negative & 106 & 46 & 60 & \\
\hline Positive & 31 & 25 & 6 & \\
\hline
\end{tabular}

Chi-square test. ${ }^{*} p<0.05$
UM-UC-3 cells expressed the lowest amount of circZKSCAN1 and EJ, 5637 expressed the highest level of circ-ZKSCAN1, they were chosen for further study. We first overexpressed circ-ZKSCAN1 in UM-UC-3 and T24, and knockdowned circ-ZKSCAN1 in EJ and 5637. qPCR analysis showed overexpression or knockdown efficiency of circ-ZKSCAN1 and confirmed that the transfection had no influence on the parental ZKSCAN1 gene (Fig. 4a, Additional file 4 Figure S2a). Compared to the negative control (NC) group, MTS assays revealed that the viability of UM-UC-3 and T24 decreased in the overexpressed group (Fig. 4b, c). Moreover, colony formation assays showed that proliferative abilities of UM-UC-3 and T24 were also inhibited in the overexpressed group (Fig. 4d, e). Furthermore, Flow cytometry analysis was performed to detect whether circ-ZKSCAN1 could affect BCa cells phenotype by altering the cell cycle. As shown in Fig. $4 \mathrm{f}, \mathrm{g}$ and $\mathrm{h}$, after overexpression of circ-ZKSCAN1, more cells were distributed in G1 phase, which suggested that circ-ZKSCAN1 might induce G1/S cell cycle arrest. Additionally, transwell migration and invasion demonstrated that the number of cells was fewer in the overexpressed group than in the NC group (Fig. 4i, j).

In contrast, knocking down circ-ZKSCAN1 promoted the proliferation, invasion and migration of $\mathrm{BCa}$ cells (Additional file 4 Figure S2 b-d). Collectively, these data 

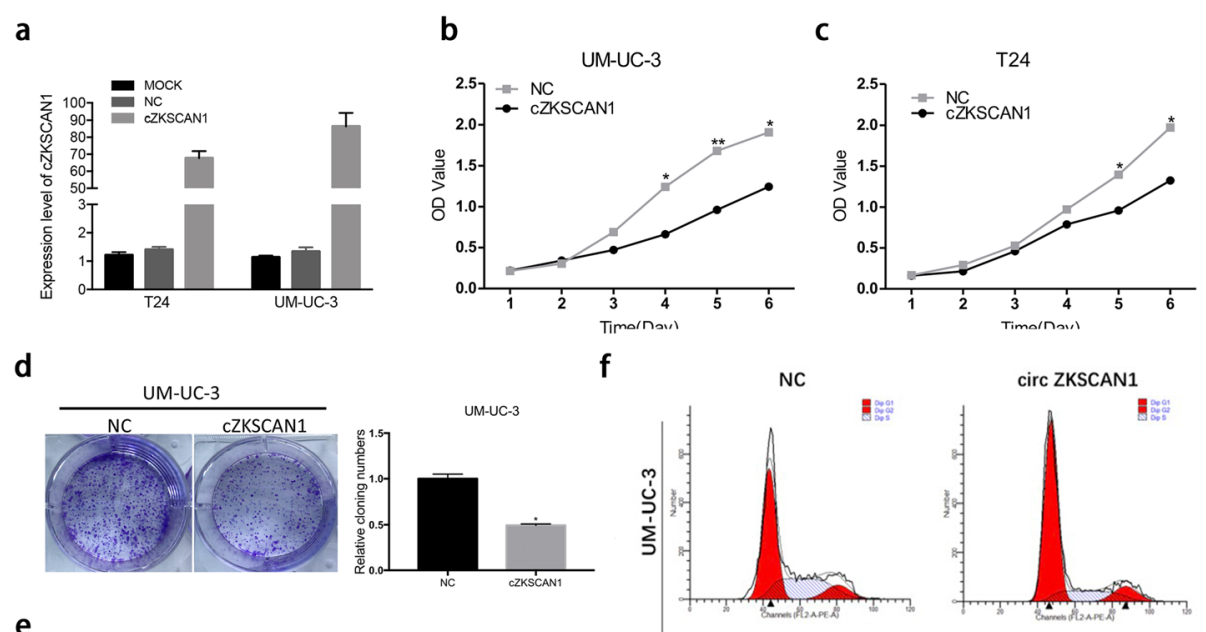

e
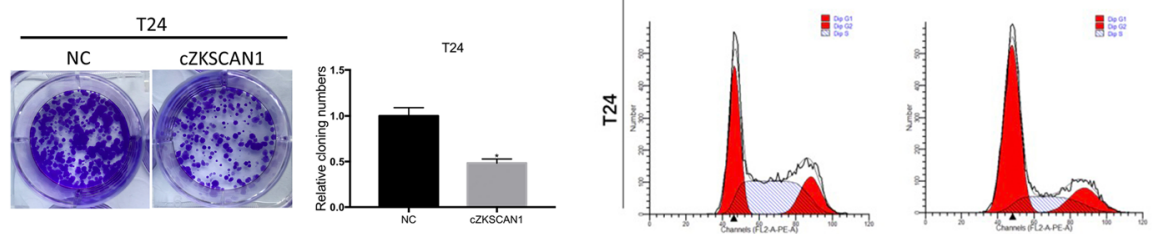

g
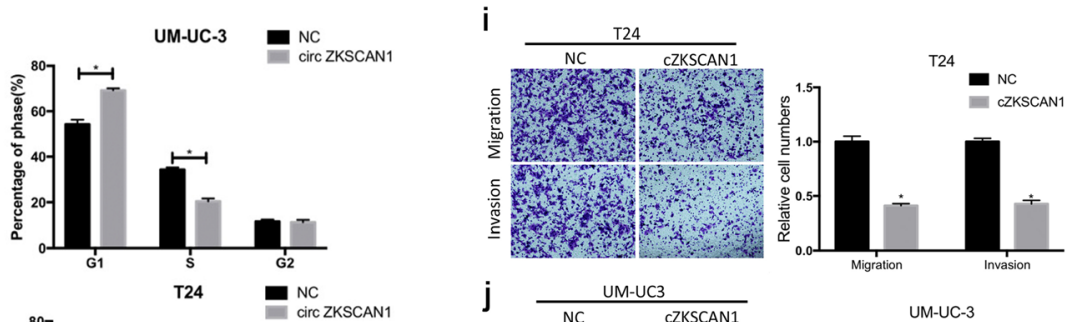

$\mathrm{h}$
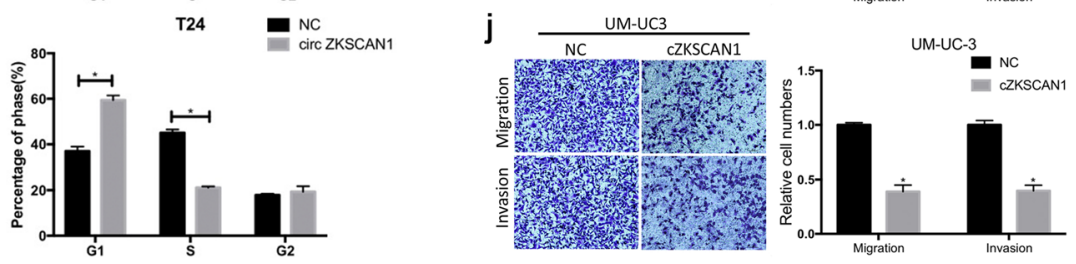

Fig. 4 Enforced expression of Circ-ZKSCAN1 inhibited proliferation, migration, and invasion of BCa cells in vitro. a After the overexpression of circZKSCAN1, QPCR was used to detect expression of circ-ZKSCAN1 in T24 and UM-UC-3. b-c MTS assays showed that circ-ZKSCAN1 could inhibite the viablities of T24 and UM-UC-3. d-e Clony formation assays demonstrated that circ-ZKSCAN1 could inhibit the formation of clonies in T24 and UMUC-3. $\mathbf{f}$ Representative images of the cell cycle analysis by using flow cytometry. $\mathbf{g}$-h Circ-ZKSCAN1 arrested the UM-UC-3 and T24 cell cycle at the G1/S phase. i-j Cell migration and invasion assays were performed and measured in T24 and UM-UC-3 transfected with circ-ZKSCAN1 or vector

suggested that enforced circ-ZKSCAN1 could inhibit the progression of $\mathrm{BCa}$ cells in vitro.

\section{Circ-ZKSCAN1 may function as a sponge of miR-1178-3p}

Given that circ-ZKSCAN1 is located in the cytoplasm, we attempted to determine whether circZKSCAN1 could sponge miRNAs. According to the microRNA response element (MRE) analysis, we used miRanda and TargetScan prediction tool to identify 12 miRNAs that could bind to circZKSCAN1 (Fig. 5a). Next, we used a biotin-coupled probe pull-down assay to confirm this prediction. The biotinylated circ-ZKSCAN1 probe and oligo probe were designed and were applied to perform
RNA pull-down assay. The pull-down efficiency was verified in UM-UC-3 cells transfected with circZKSCAN1 or vector. As shown in Fig. 5b, the specific enrichments of miR-1178-3p, miR-29b-3p and miR-765 that were detected in the circ-ZKSCAN1 pull-down pellet was significantly higher than NC group.

To further confirm this result, we conducted a luciferase assay using mimics of miR-1178-3p, miR29b-3p and miR-765 (Additional file 5 Figure S3a). We co-transfected each mimics and a luciferase reporter containing sequence of circ-ZKSCAN1 into HEK293T cells. Compared to the NC, only miR$1178-3 p$ could significantly reduce the luciferase 


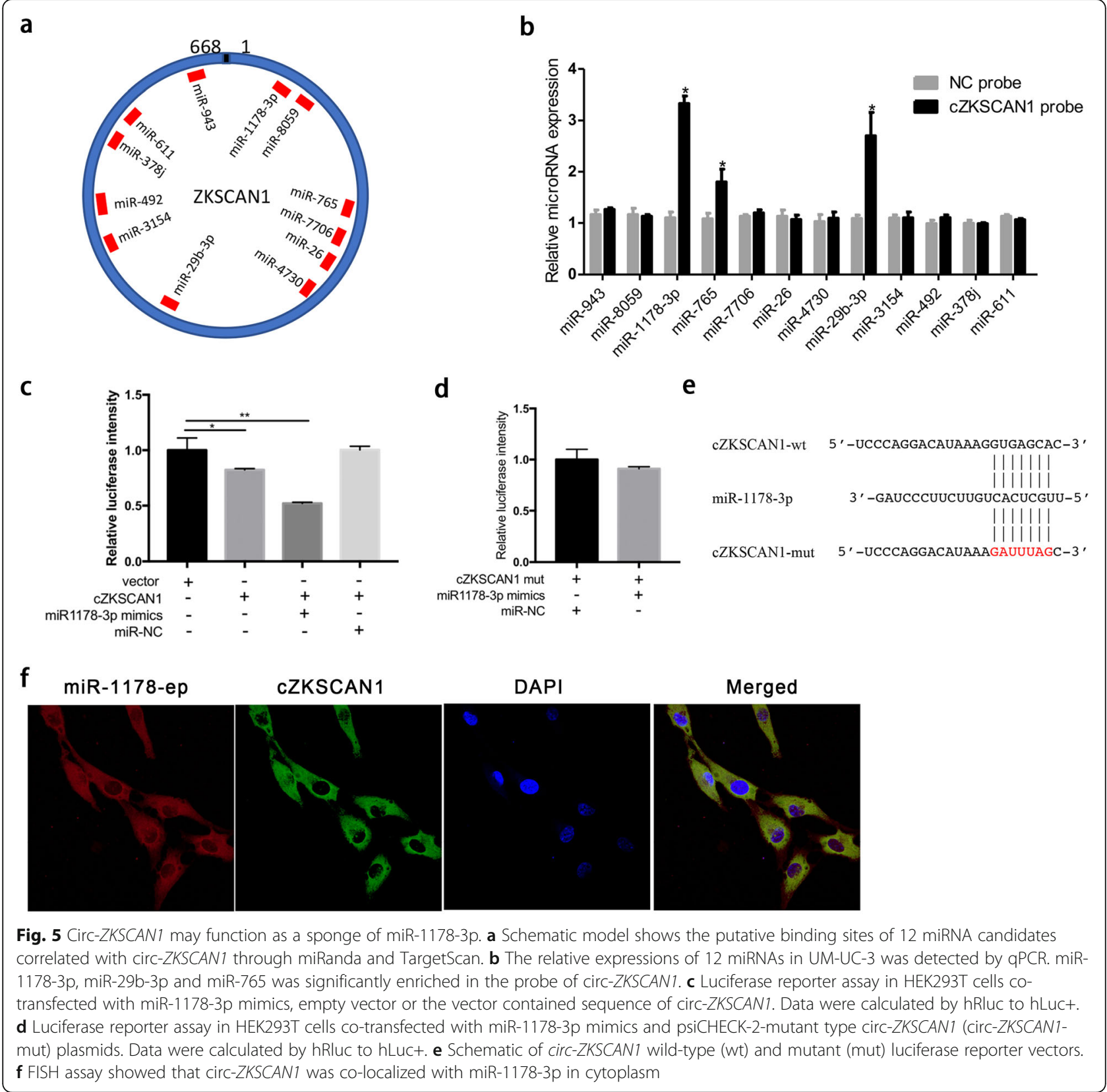

activity (Fig. 5c). Additionally, we found that the cotransfected miR-1178-3p mimics and mutant reporter had no effect on luciferase activity (Fig. 5d, e). We further performed FISH assay to confirm the co-localization of circ-ZKSCAN1 and miR-1178-3p in the cytoplasm (Fig. 5f). Collectively, these results implied that circ-ZKSCAN1 could function as a sponge through targeting miR-1178-3p.

\section{MiR-1178-3p exerts an oncogenic role and partially} alleviates effect of circ-ZKSCAN1 in BCa

MiR-1178-3p is an oncogene in baldder cancer reported by Liu et al. [26]. In the present study, we found that the expression of miR-1178-3p was upregulated in 56 pairs of $\mathrm{BCa}$ tissues compared to the adjacent normal tissues (Additional file 6 Figure S4a). Furthermore, miR-1178-3p was also upregulated in $\mathrm{BCa}$ cell lines (Additional file 6 Figure S4b). After the transfection mimics of miR-1178-3p into T24 and UM-UC-3, the abilities of migration, invasion and proliferation were significantly enhanced (Fig. 6a, b). Moreover, rescue experiments were conducted by co-transfecting miR1178-3p mimics and circ-ZKSCAN1 in T24 and UMUC-3. Remarkably, miR-1178-3p mimics could partially alleviate the tumor-suppressing effect of circ-ZKSCAN1 (Fig. 6c, d, e, f, g). 
a

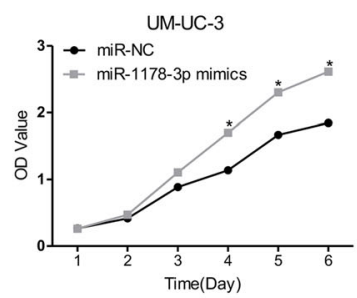

b

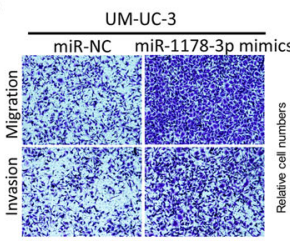

C

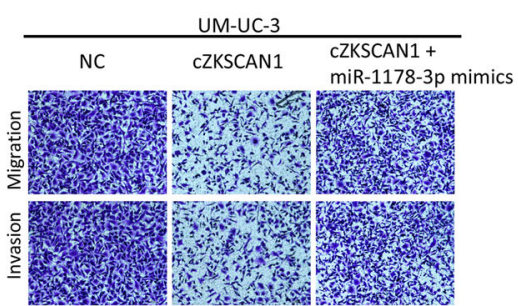

UM-UC-3

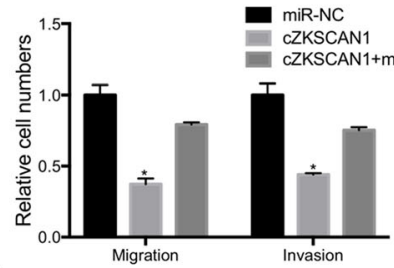

d

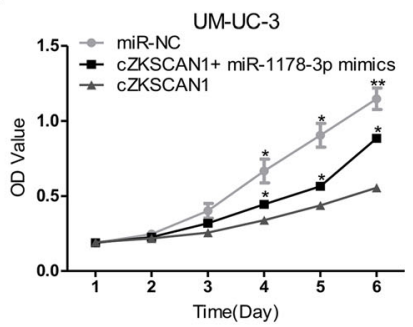

e

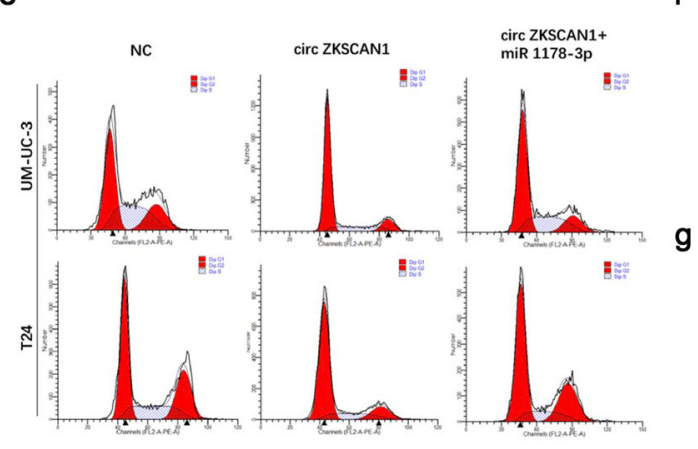

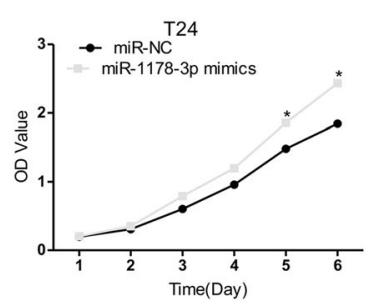
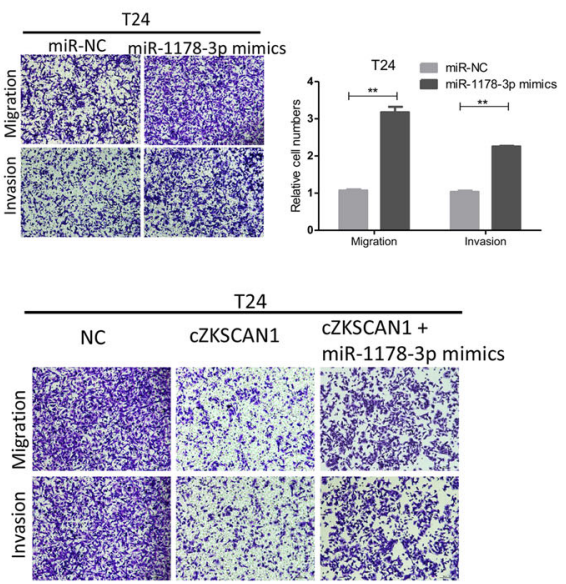

$\mathrm{T} 24$

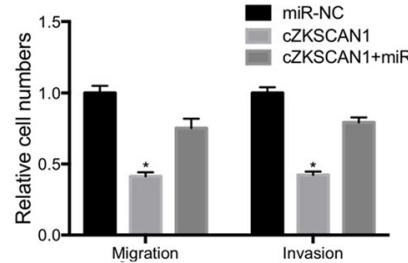

$\mathrm{T} 24$

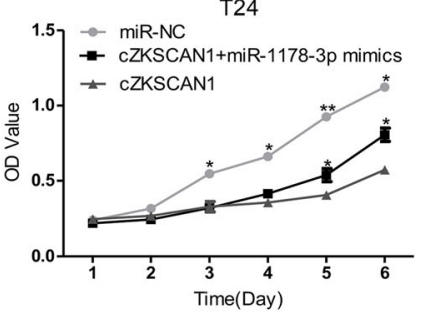

f
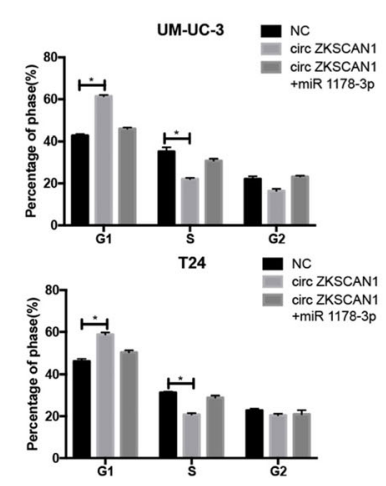

Fig. 6 (See legend on next page.) 
(See figure on previous page.)

Fig. 6 MiR-1178-3p exerts an oncogenic role and partly alleviates effect of circ-ZKSCAN1 in BCa. a-b Transfection of miR-1178-3p mimics enhace the abilities of proliferation, migration and invasion in UM-UC-3 and T24. c-d miR-1178-3p could partially reverse the anti-oncogenic effects of circ-ZKSCAN1 on migration, invasion and proliferation in UM-UC-3 and T24. e Representative images of the cell cycle analysis by using flow cytometry. f-g Circ-ZKSCAN1 arrested the UM-UC-3 and T24 cell cycle at the G1/S phase, while miR-1178-3p could partially turned the G1/S transition

Overall, these results suggest that circ-ZKSCAN1 may inhibit $\mathrm{BCa}$ cells progression partially through impairing the oncogenic role of miR-1178-3p.

\section{Circ-ZKSCAN1 downregulates p21 expression by partly sponging miR-1178-3p}

To explore the molecular mechanism of circ-ZKSCAN1 in $\mathrm{BCa}$, we performed a RNA-seq analysis. Among all genes that are regulated by circ-ZKSCAN1, multiple genes play a vital role in the proliferation and invasion of $\mathrm{BCa}$. As shown in Fig. 7a, p21 (CDKN1A) was significantly downregulated in circ-ZKSCAN1-silenced cells. To further confirm this result, qPCR and western blotting were performed. The expression of $\mathrm{p} 21$ at both the mRNA and protein levels was upregulated in circ-ZKSCAN1-overexpressed cells. In total (Fig. 7b, c), these results indicated that circ-ZKSCAN1 suppressed the proliferation and invasion of $\mathrm{BCa}$ cells through regulation of $\mathrm{p} 21$.

According to the MRE analysis, miR-1178-3p might target p21 (Fig. 7d). After the transfection of miR-1178$3 p$ mimics, the expression of p21 was significantly reduced at the protein level (Fig. 7e). Additionally, a dual luciferase reporter assay was performed. The wild-type (WT) 3'-UTR sequence of p21 was cloned into the pcDNA3 vector. Co-transfection of miR-1178-3p mimics and p21 wild-type (wt) plasmids strongly reduced luciferase activity by over $41 \%$ (Fig. $7 \mathrm{f}$ ). However, co-transfection of p21 the mutated vector and miR1178-3p mimics had no obvious effect on luciferase activity (Fig. 7f). Moreover, miR-1178-3p could partially rescue the effect of circ-ZKSCAN1 on the expression of p21 (Fig. 7g).

Taken together, these results revealed that circ-ZKSCAN1 suppressed the progression of $\mathrm{BCa}$ partially through the miR-1178-3p /p21 axis.

\section{Overexpression of Circ-ZKSCAN1 suppresses proliferation and invasion of $\mathrm{BCa}$ cells in vivo}

To explore the effects of circ-ZKSCAN1 on BCa tumorigenesis in vivo, circ-ZKSCAN1 was overexpressed in UM-UC-3. Cell from the circ-ZKSCAN1-overexpressed and NC groups were subcutaneously injected into $\mathrm{BALB} / \mathrm{c}$ nude mice, and the tumor volumes were measured weekly (Fig. 8a, b). The sizes and weights of the tumors from the circ-ZKSCAN1-overexpressed group were significantly smaller than those of the control group (Fig. 8c, d). These subcutaneous tumors were further subjected to immunohistochemical staining. The expression of p21 was significantly upregulated in the circ-ZKSCAN1-overexpressed group compared to the $\mathrm{NC}$ group (Fig. 8e). To investigate the role of circZKSCAN1 in tumor metastasis, circ-ZKSCAN1-overexpressed/luc cells and wild-type/luc cells were injected into the foot pads of BALB/C nude mice. The bioluminescence of the popliteal LNs was either undetectable in the circ-ZKSCAN1-overexpressed group or dramatically lower than in the $\mathrm{NC}$ group (Fig. 8f and g). Western blotting was applied to detect the protein expression of p21 in xenografted tumors of each group. Compare to the NC group,the expression of p21 was obviously increased in tumor tissues of circ-ZKSCAN1-overexpressed group (Fig. 8h). Taken together, these data indicated that circ-ZKSCAN1 inhibited the progression of $\mathrm{BCa}$ in vivo.

\section{Discussion}

Currently, a large class of circRNAs have been identified by massively parallel sequencing technology [7]. Previous studies revealed that the expression of circRNAs is cell type- specific and tissue-specific and circRNAs have been proven to be potential biomarkers [8]. CircRNAs are involved in the progression of many cancers by being sponges of microRNAs and keeping target genes away from miRNAs [9-11]. However, the functions of circRNAs in BCa remain largely unkown. In this study, we first analyzed and selected diferentially expressed circRNAs from microarray data [9-11]. Then, we established a BCa cell invasion model and screened invasion related circRNAs. Among the aberrantly expressed circRNAs, circ-ZKSCAN1 was significantly upregulated in normal tissues and lowly invasive cell subline. Circ-ZKSCAN1 was first reported by Liang and Wilusz, who showed that circZKSCAN1 was partially abundant in human brain and liver [27]. Yang found that circ-ZKSCAN1 was downregulated in hepatocellular carcinoma and acted as a tumor suppressor [28]. To our knowledge, this is the first report on the expression and regulatory function of circZKSCAN1 in BCa.

Interestingly, the expression of circ-ZKSCAN1 was dramatically downregulated not only in $\mathrm{BCa}$ but also in $\mathrm{BCa}$ cell lines. Additionally, we found that lower expression of circ-ZKSCAN1 was significantly associated with poorer disease free survival, higher recurrence and a positive tumor metastasis status, suggesting its tumor-suppressive effect on 
a

d
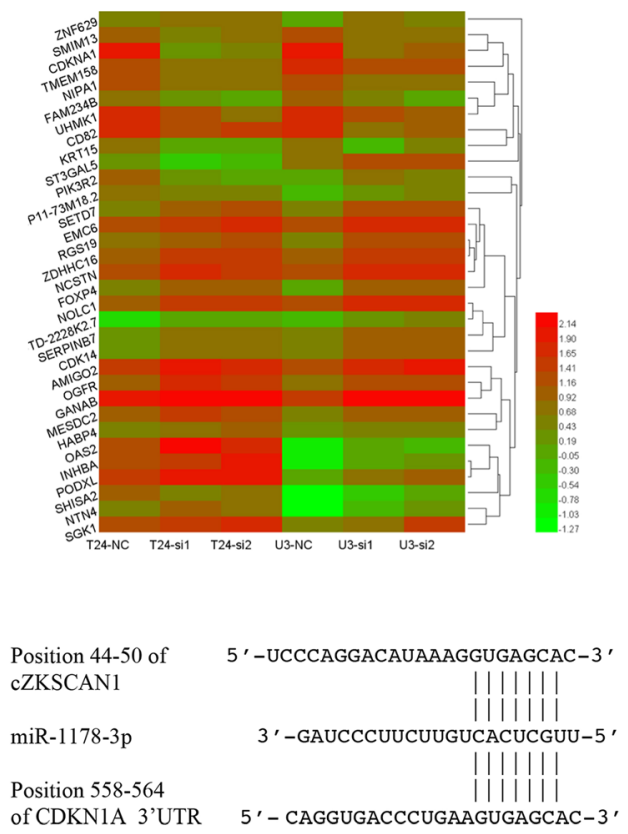
of CDKN1A 3'UTR

f

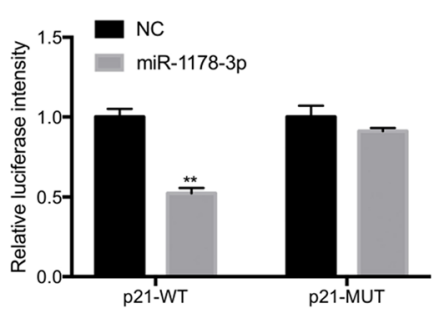

g b

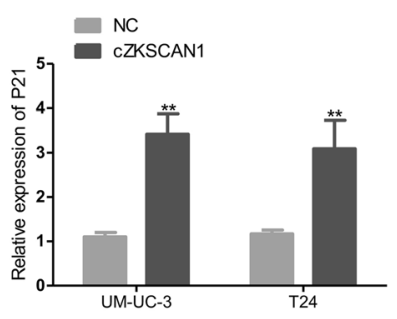

C

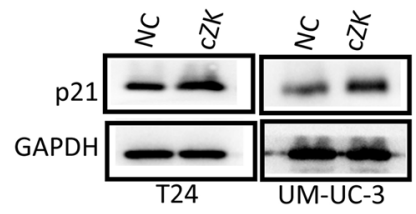

e

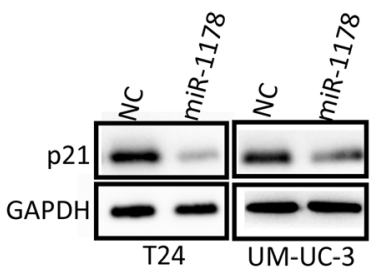

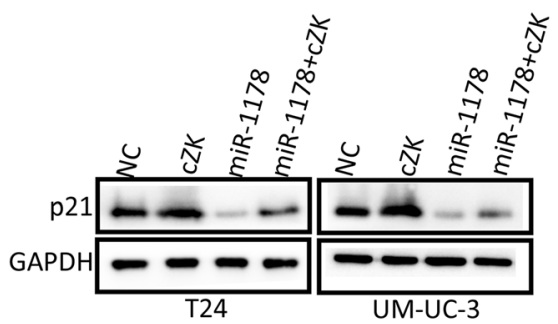

Fig. 7 Circ-ZKSCAN1 downregulates p21(CDKN1A) expression by partially sponging miR-1178-3p. a Differentially expressed genes between the Negative Control (NC) and siRNAs groups were showed in the heatmap. Red indicates upregulated expression; green indicates downregulated expression. b-c qPCR and western blotting showed that enforced circ-ZKSCAN1(cZK) increased the expression of p21(CDKN1A) at both the mRNA and protein levels in UM-UC-3 and T24. $\mathbf{d}$ The schematics revealed that circ-ZKSCAN1(cZK) and p21 shared the same binding sites of miR-1178-3p. e Western blotting showed that p21 was downregulated in UM-UC-3 and T24 after transfection of miR-1178-3p mimics. f The dual-luciferase reporter assays revealed that luciferase activities was decreased in HEK293T cells after co-transfection with miR-1178-3p mimics and p21-wild-type (p21-wt) vector or p21-mutant (p21-mut) vector. g p21 protein expression levels were detected by western blotting after transfected miR-1178-3p mimics or co-transfected miR-1178-3p and circ-ZKSCAN1(cZK). The abbreviations of circ-ZKSCAN1, miR-1178-3p mimics and CDKN1A are cZK, miR1178 and p21, respectively

BCa. In functional studies, cell proliferation, clone formation, migration and invasion were significantly inhibited in the circ-ZKSCAN1-overexpressed group compared with the NC group. Moreover, to explore the effects of circZKSCAN1 in vivo, circ-ZKSCAN1-overexpressed UM-UC3 and wild-type UM-UC-3 cells were injected into the back and foot pads of nude mice. Strikingly, the growth and metastasis of tumors from circ-ZKSCAN1-overexpressed UM-UC-3 were significantly inhibited. Taken together, these findings indicate that circ-ZKSCAN1 is a tumor suppressor in $\mathrm{BCa}$.

An increasing number of circRNAs that possess the ability to bind to miRNAs has been reported. For example, circ-ITCH binds to miR-17 in colorectal cancer and lung cancer [14]. Another circRNA termed circ-SMARCA5 was observed to be a sponge for miR-17 and miR-181b [11]. In our study, we used the miRanda and TargetScan prediction tool to screen oncogenic miRNAs. Finally, through a biotin-coupled probe pull-down assay and dual luciferase assay, miR-1178-3p was selected and confirmed to be the target miRNA of circ-ZKSCAN1. MiR-1178-3p, an oncogene reported by Cao Z. et al., targets tumor suppressors in pancreatic cancer [29]. Further experiments have confirmed that miR-1178-3p promotes the progression of $\mathrm{BCa}$ and is upregulated in $\mathrm{BCa}$. To explore the underlying mechanism by which circ-ZKSCAN1 regulates proliferation and invasion in $\mathrm{BCa}$, we performed RNA-seq to identify changes in gene expression after silencing circ- 
a

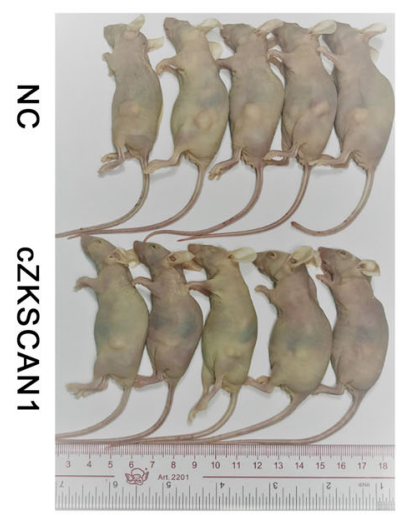

C

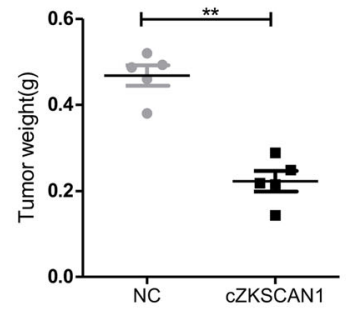

e

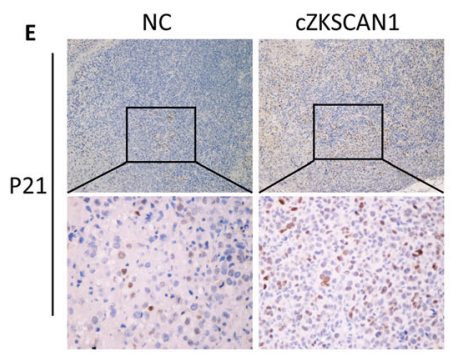

f

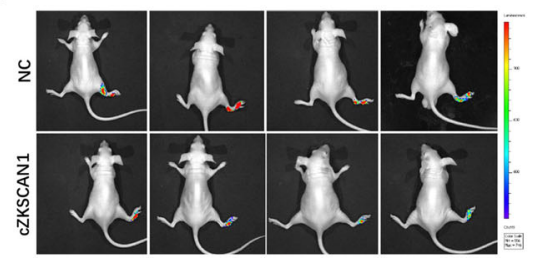

b

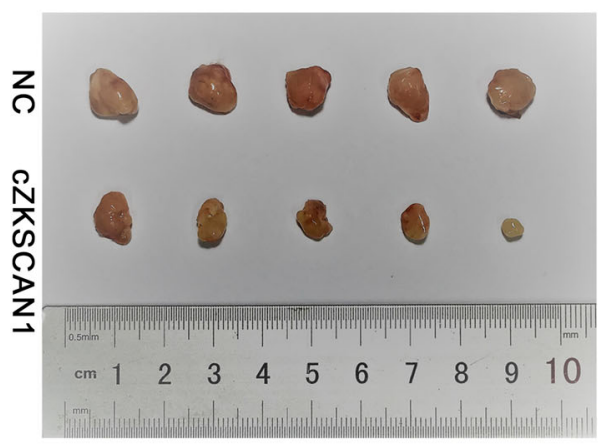

d
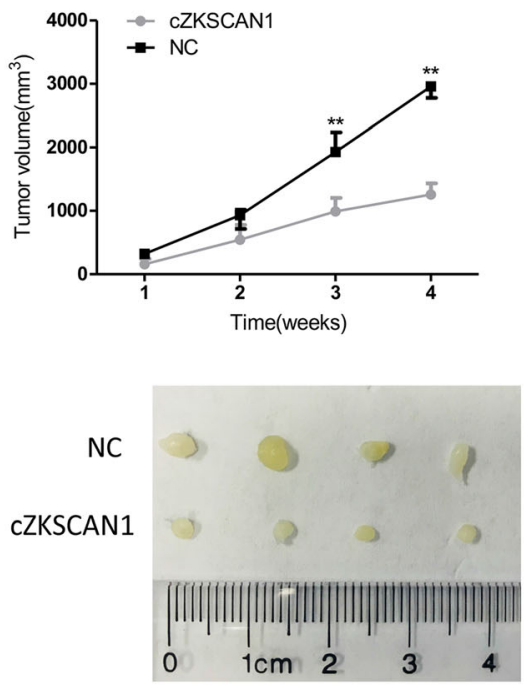

h

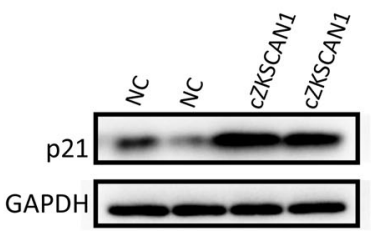

Fig. 8 Overexpression of Circ-ZKSCAN1 Suppresses Proliferation Growth and Invasion of BCa cells in vivo. a-d UM-UC-3 stably expressed circZKSCAN1 or wild-type UM-UC-3 were injected into BALB/C nude mice. ( $n=5$ for each group) Compare with NC group, tumor weight and volume significantly reduced in overexpressed group. e IHC revealed that the expression of p21 significantly enhanced in overexpressed group, compare to NC group. $\mathbf{f}-\mathbf{g}$ The luciferase images demonstrated that enforced circ-ZKSCAN1 could inhibited metastasis in the foot pads model. The popliteal lymph nodes was smaller in overexpressed group than in the NC group. ( $n=4$ for each group) $\mathbf{h}$ Western blotting revealed that overexpression of circ-ZKSCAN1 led to increased expression of p21 within xenografted tumors

ZKSCAN1. The expression of p21 changed significantly and was confirmed by qPCR and western blot. As a wellknown tumor suppressor, p21 can inhibit the growth and metastasis of multiple cancer types [24]. Therefore, we predicted that p21 might be the target gene of circZKSCAN1 and miR-1178-3p. According to the bioinformatics analysis, dual luciferase reporter assays and rescue experiments, we verified a novel regulatory axis that was formed by circ-ZKSCAN1/miR-1178-3p/p21 in BCa.

\section{Conclusion}

In summary, we first analyzed and selected circRNAs from circRNA microarray. Then, we have established $\mathrm{BCa}$ cell invasion model and found that circ-ZKSCAN1 is significantly downregulated in $\mathrm{BCa}$ tissues and highly invasive $\mathrm{BCa}$ cell lines. This is the first study to explore the profile and mechanism of circ-ZKSCAN1 in BCa. Circ-ZKSCAN1 is downregulated in $\mathrm{BCa}$ and correlates with tumor metastasis status, recurrence, pathological $\mathrm{T}$ stage and histological 
grade. Additionally, patients with lower circ-ZKSCAN1 expressions might have poorer prognosis. In functional and mechanistic assays, circ-ZKSCAN1 suppresses BCa progression in vivo and in vitro through a novel circ-ZKSCAN1/ $\mathrm{miR}-1178-3 \mathrm{p} / \mathrm{p} 21$ signaling regulatory network. Taken together, these data indicated that circ-ZKSCAN1 might be a potential biomarker and prognostic factor for $\mathrm{BCa}$.

\section{Additional files}

Additional file 1: Table S1 The sequences of primers, oligonucleotides and probes used in this study. (PDF $47 \mathrm{~kb}$ )

Additional file 2: Table S2 Identification of differentially expressed circRNAs in BCa cells (PDF $10 \mathrm{~kb}$ )

Additional file 3: Figure S1 Data from TCGA and our cohort showed the expression of ZKSCAN1 mRNA between BCa tissues and paired adjacent normal tissues. a Data from TCGA revealed that there was no significant changes in ZKSCAN1 mRNA between BCa tissues and matched adjacent normal tissues. $\mathbf{b}$ qPCR analysis for ZKSCAN1 mRNA in our cohort 1. (TIF $9877 \mathrm{~kb}$ )

Additional file 4: Figure S2 circ-ZKSCAN1 silencing promotes proliferation, migration and invasion of BCa cells in vitro. a $\mathrm{qPCR}$ analysis for circ-ZKSCAN1 and ZKSCAN1 mRNA in EJ and 5637 cells treated with siRNAs or transfected with negative control. b-c The effect of si- circZKSCAN1 on cell proliferation of EJ and 5637 cells was assessed by colony formation assays. $\mathbf{d}$-e The cell migratory and invasive capabilities were examined in EJ and 5637 cells treated with circ-ZKSCAN1 siRNAs using transwell migration and invasion assays. (TIF $11349 \mathrm{~kb}$ )

Additional file 5: Figure S3 Circ-ZKSCAN1 may function as a sponge of miR-1178-3p a The Renilla luciferase activity of wild type circ-ZKSCAN1 in the miR-29b-3p/miR-765 mimics or NC group. (TIF $3244 \mathrm{~kb}$ )

Additional file 6: Figure S4 MiR-1178-3p exerts an oncogenic role in BCa a-b qPCR revealed that miR-1178-3p was up-regulated in BCa tissues $(n=56)$ and cell lines (UM-UC-3 and T24), compared to the paired normal bladder tissues and SV-HUC-1. (JPG $447 \mathrm{~kb}$ )

\section{Abbreviations}

BCa: Bladder cancer; CircRNAs: Circular RNAs; CZKSCAN1(cCK): circular RNA ZKSCAN1; LN: Lymph node; M: Highly invasive cell line; MRE: microRNA response element; NM: Lowly invasive cell line; p21 (CDKN1A): cyclindependent kinase inhibitor 1A; qPCR: quantitative real-time polymerase chain reaction; ZKSCAN1: Zinc finger with KRAB and SCAN domains 1

\section{Acknowledgements}

Not applicable.

\section{Authors' contributions}

$J \mathrm{H}$ and TXL conceived of the study and carried out its design. JMB, HWL, WD, and WBX performed the experiments. JMB, ZJC, and LHW conducted the statistical analyses. JMB wrote the paper and WD revised the paper. All authors read and approved the final manuscript.

\section{Funding}

This work was supported by grants from the National Natural Science Foundation of China (Grant NO. 81772728, 81772719); Guangdong Science and Technology Development Fund (2017B020227007).

\section{Availability of data and materials}

The circRNA microarray data of BCa tissues and matched normal tissues analysed during this study are included in this published article and its supplementary information files (PMID: 27050392, PMCID: PMC4823868, https://doi.org/10.1038/ncomms11215). The rest of datasets used and/or analysed during the current study are available from the corresponding author on reasonable request.

\section{Ethics approval and consent to participate}

The present study was approved by the Medical Ethics Committee of Sun Yat-sen Memorial Hospital and Experimental animal ethics committee of Sun Yat-sen University.

\section{Consent for publication}

Not applicable.

\section{Competing interests}

The authors declare that they have no competing interests.

\section{Author details}

${ }^{1}$ Department of Urology, Sun Yat-Sen Memorial Hospital, Sun Yat-Sen University, 107.W. Yanjiang Road, Guangzhou, Guangdong 510120, People's Republic of China. ${ }^{2}$ Guangdong Provincial Key Laboratory of Malignant Tumor Epigenetics and Gene Regulation, Sun Yat-Sen Memorial Hospital, Sun Yat-Sen University, Guangzhou, People's Republic of China. ${ }^{3}$ Department of Urology, Affiliated Hospital of Guangdong Medical University, Zhanjiang, People's Republic of China. ${ }^{4}$ Department of Urology, Union Hospital, Tongji Medical College, Huazhong University of Science and Technology, Wuhan, People's Republic of China.

Received: 23 June 2019 Accepted: 19 August 2019

Published online: 03 September 2019

\section{References}

1. Torre LA, Bray F, Siegel RL, Ferlay J, Lortet-Tieulent J, Jemal A. Global cancer statistics, 2012. CA Cancer J Clin. 2015;65:87-108.

2. Wu XR. Urothelial tumorigenesis: a tale of divergent pathways. Nat Rev Cancer. 2005:5:713-25.

3. Hautmann RE, de Petriconi RC, Pfeiffer C, Volkmer BG. Radical cystectomy for urothelial carcinoma of the bladder without neoadjuvant or adjuvant therapy: long-term results in 1100 patients. Eur Urol. 2012;61:1039-47.

4. Strilic B, Offermanns S. Intravascular survival and extravasation of tumor cells. Cancer Cell. 2017;32:282-93.

5. Jeck WR, Sharpless NE. Detecting and characterizing circular RNAs. Nat Biotechnol. 2014:32:453-61.

6. Lasda E, Parker R. Circular RNAs: diversity of form and function. Rna. 2014;20: 1829-42.

7. Memczak S, Jens M, Elefsinioti A, Torti F, Krueger J, Rybak A, Maier L, Mackowiak SD, Gregersen LH, Munschauer M, et al. Circular RNAs are a large class of animal RNAs with regulatory potency. Nature. 2013;495:333-8.

8. Guo JU, Agarwal V, Guo H, Bartel DP. Expanded identification and characterization of mammalian circular RNAs. Genome Biol. 2014;15:409.

9. Zhang J, Liu H, Hou L, Wang G, Zhang R, Huang Y, Chen X, Zhu J. Circular RNA_LARP4 inhibits cell proliferation and invasion of gastric cancer by sponging miR-424-5p and regulating LATS1 expression. Mol Cancer. 2017; 16:151.

10. He Q, Zhao L, Liu Y, Liu X, Zheng J, Yu H, Cai H, Ma J, Liu L, Wang P, et al. Circ-SHKBP1 regulates the angiogenesis of U87 glioma-exposed endothelial cells through miR-544a/FOXP1 and miR-379/FOXP2 pathways. Mol Ther Nucleic Acids. 2018;10:331-48.

11. Yu J, Xu QG, Wang ZG, Yang Y, Zhang L, Ma JZ, Sun SH, Yang F, Zhou WP. Circular RNA CSMARCA5 inhibits growth and metastasis in hepatocellular carcinoma. J Hepatol. 2018;68:1214-27.

12. Beermann J, Piccoli MT, Viereck J, Thum T. Non-coding RNAs in development and disease: background, mechanisms, and therapeutic approaches. Physiol Rev. 2016;96:1297-325.

13. Thomson DW, Dinger ME. Endogenous microRNA sponges: evidence and controversy. Nat Rev Genet. 2016;17:272-83.

14. Yang C, Yuan W, Yang X, Li P, Wang J, Han J, Tao J, Li P, Yang H, LV Q, Zhang W. Circular RNA circ-ITCH inhibits bladder cancer progression by sponging miR-17/miR-224 and regulating p21, PTEN expression. Mol Cancer. 2018;17:19

15. Li Y, Zheng F, Xiao X, Xie F, Tao D, Huang C, Liu D, Wang M, Wang L, Zeng F, Jiang G. CircHIPK3 sponges miR-558 to suppress heparanase expression in bladder cancer cells. EMBO Rep. 2017;18:1646-59.

16. Allione A, Pardini B, Viberti C, Giribaldi G, Turini S, Di Gaetano C, Guarrera S, Cordero F, Oderda M, Allasia M, et al. MMP23B expression and protein levels in blood and urine are associated with bladder cancer. Carcinogenesis. 2018:39:1254-63. 
17. Liu X, Liu X, Wu Y, Fang Z, Wu Q, Wu C, Hao Y, Yang X, Zhao J, Li J, et al. MicroRNA-34a attenuates metastasis and Chemoresistance of bladder Cancer cells by targeting the TCF1/LEF1 Axis. Cell Physiol Biochem. 2018;48:87-98.

18. van Kampen J, van Hooij O, Jansen CF, Smit FP, van Noort PI, Schultz I, Schaapveld R, Schalken JA, Verhaegh GW. miRNA-520f reverses epithelial-tomesenchymal transition by targeting ADAM9 and TGFBR2. Cancer Res. 2017; 77:2008-17.

19. Dyrskjot L, Ostenfeld MS, Bramsen JB, Silahtaroglu AN, Lamy P, Ramanathan R, Fristrup N, Jensen JL, Andersen CL, Zieger K, et al. Genomic profiling of microRNAs in bladder cancer: miR-129 is associated with poor outcome and promotes cell death in vitro. Cancer Res. 2009:69:4851-60.

20. Chen LL. The biogenesis and emerging roles of circular RNAs. Nat Rev Mol Cell Biol. 2016;17:205-11.

21. Tie J, Pan Y, Zhao L, Wu K, Liu J, Sun S, Guo X, Wang B, Gang Y, Zhang Y, et al. MiR-218 inhibits invasion and metastasis of gastric cancer by targeting the Robol receptor. PLoS Genet. 2010;6:e1000879.

22. Soukup V, Capoun O, Cohen D, Hernandez V, Babjuk M, Burger M, Comperat E, Gontero P, Lam T, MacLennan S, et al. Prognostic performance and reproducibility of the 1973 and 2004/2016 World Health Organization grading classification Systems in non-muscle-invasive Bladder Cancer: a European Association of Urology non-muscle invasive bladder Cancer guidelines panel systematic review. Eur Urol. 2017;72:801-13.

23. Sun M, Trinh QD. Diagnosis and staging of bladder cancer. Hematol Onco Clin North Am. 2015;29:205-18

24. Abbas T, Dutta A. p21 in cancer: intricate networks and multiple activities. Nat Rev Cancer. 2009:9:400-14.

25. Zheng Q, Bao C, Guo W, Li S, Chen J, Chen B, Luo Y, Lyu D, Li Y, Shi G, et al. Circular RNA profiling reveals an abundant circHIPK3 that regulates cell growth by sponging multiple miRNAs. Nat Commun. 2016;7:11215.

26. Liu H, Bi J, Dong W, Yang M, Shi J, Jiang N, Lin T, Huang J. Invasion-related circular RNA circFNDC3B inhibits bladder cancer progression through the miR-1178-3p/G3BP2/SRC/FAK axis. Mol Cancer. 2018;17:161.

27. Liang D, Wilusz JE. Short intronic repeat sequences facilitate circular RNA production. Genes Dev. 2014;28:2233-47.

28. Yao Z, Luo J, Hu K, Lin J, Huang H, Wang Q, Zhang P, Xiong Z, He C, Huang $Z$, et al. ZKSCAN1 gene and its related circular RNA (circZKSCAN1) both inhibit hepatocellular carcinoma cell growth, migration, and invasion but through different signaling pathways. Mol Oncol. 2017;11:422-37.

29. Cao Z, Xu J, Huang H, Shen P, You L, Zhou L, Zheng L, Zhang T, Zhao Y. MiR-1178 promotes the proliferation, G1/S transition, migration and invasion of pancreatic cancer cells by targeting CHIP. PLoS One. 2015;10:e116934.

\section{Publisher's Note}

Springer Nature remains neutral with regard to jurisdictional claims in published maps and institutional affiliations.

Ready to submit your research? Choose BMC and benefit from:

- fast, convenient online submission

- thorough peer review by experienced researchers in your field

- rapid publication on acceptance

- support for research data, including large and complex data types

- gold Open Access which fosters wider collaboration and increased citations

- maximum visibility for your research: over $100 \mathrm{M}$ website views per year

At $\mathrm{BMC}$, research is always in progress.

Learn more biomedcentral.com/submissions 